\title{
Challenges of Modeling Outcomes for Surgical Infections: A Word of Caution
}

\author{
Fabian Grass, ${ }^{1}$ Curtis B. Storlie, ${ }^{2}$ Kellie L. Mathis, ${ }^{1}$ John R. Bergquist ${ }^{1,2}$ Shusaku Asai, ${ }^{3}$ \\ Judy C. Boughey, ${ }^{1}$ Elizabeth B. Habermann, ${ }^{2}$ David A. Etzioni, ${ }^{4}$ and Robert R. Cima ${ }^{1}$
}

\begin{abstract}
Background: We developed a novel analytic tool for colorectal deep organ/space surgical site infections (COSI) prediction utilizing both institutional and extra-institutional American College of Surgeons-National Surgical Quality Improvement Program (ACS-NSQIP) data.

Methods: Elective colorectal resections (2006-2014) were included. The primary end point was C-OSI rate. A Bayesian-Probit regression model with multiple imputation (BPMI) via Dirichlet process handled missing data. The baseline model for comparison was a multivariable logistic regression model (generalized linear model; GLM) with indicator parameters for missing data and stepwise variable selection. Out-of-sample performance was evaluated with receiver operating characteristic (ROC) analysis of 10-fold cross-validated samples.

Results: Among 2,376 resections, C-OSI rate was 4.6\% $(\mathrm{n}=108)$. The BPMI model identified $(\mathrm{n}=57 ; 56 \%$ sensitivity) of these patients, when set at a threshold leading to $80 \%$ specificity (approximately a $20 \%$ false alarm rate). The BPMI model produced an area under the curve (AUC) $=0.78$ via 10 -fold cross- validation demonstrating high predictive accuracy. In contrast, the traditional GLM approach produced an AUC $=0.71$ and a corresponding sensitivity of 0.47 at $80 \%$ specificity, both of which were statstically significant differences. In addition, when the model was built utilizing extra-institutional data via inclusion of all (non-Mayo Clinic) patients in ACS-NSQIP, C-OSI prediction was less accurate with AUC $=0.74$ and sensitivity of 0.47 (i.e., a 19\% relative performance decrease) when applied to patients at our institution.

Conclusions: Although the statistical methodology associated with the BPMI model provides advantages over conventional handling of missing data, the tool should be built with data specific to the individual institution to optimize performance.
\end{abstract}

Keywords: colorectal; modeling; organ space infection; risk prediction

A CCURATE PREDICTION of complications before their actual occurrence is an efficient way to decrease morbidity and reduce treatment-related costs $[1,2]$. A variety of predictive risk models are available in colorectal surgery, aiming to provide guidance for early detection and management of complications $[3,4]$. However, many models are of limited accuracy given the complexity of multifactorial outcomes. Colorectal surgical site infections (C-SSI) are a major source of post-operative morbidity $[5,6]$. Hence, reduction of morbidity and the associated cost of C-SSI make mitigation a frequent focus of quality improvement initiatives with implications for surgeons, hospitals, the government, and payers [7-9]. Although some drivers of SSI incidence are universal
$[9,10]$, others are institution-specific [11-13]. A multitude of C-SSI predictive models are available, however, they have limited predictive capability when applied to independent datasets because of inconsistencies in data acquisition and management [11]. Although there are a number of predictive models to assess SSI risk in the pre- and post-operative setting [14], accurate early identification of patients who will develop C-SSI remains challenging $[15,16]$. Furthermore, the diagnosis of SSI frequently occurs after discharge in a substantial proportion of patients [17,18].

Part of the difficulty with SSI prediction is the high falsepositive rate of clinical SSI predictors including patient complaints, physical examination findings, and laboratory tests. In

\footnotetext{
${ }^{1}$ Division of Colon and Rectal Surgery, ${ }^{2}$ Department of Surgery, ${ }^{3}$ Robert D. and Patricia E. Kern Center for the Science of Health Care Delivery, Mayo Clinic, Rochester, Minnesota, USA.

${ }^{4}$ Division of Colon and Rectal Surgery, Department of Surgery, Mayo Clinic, Scottsdale, Arizona, USA.
} 
an attempt to improve predictive models of SSI in the clinical setting, the use of standardized surveillance programs have been applied to improve model performance [19]. However, the pressure to reduce costs limits the utilization of relatively specific but expensive screening tests such as computed tomography (CT) scanning [20]. Local institution-specific definitions, conditions, and standards of care confound straightforward comparisons between institutions. A recent study failed to demonstrate good interobserver performance of several different risk prediction models in independent institutional datasets [11].

A particular challenge in the use of retrospective analysis of SSI predictors is the presence of missing data [21], even in well-maintained prospective datasets [22]. Because missing data may impact retrospective analysis and prospective predictions, it is appropriate to offer a robust treatment for this problem. Prediction of SSI based on objective pre-operative and surgical risk factors may increase physicians' awareness, facilitate surveillance, and encourage early diagnostics and intervention. Given the extensive literature regarding institutional and universal SSI predictors and recent developments regarding the treatment and handling of missing data, we sought to develop a novel analytic tool for clinically relevant deep organ/space SSI and to test it in an institutional patient cohort. In a second step, the external applicability of the tool was assessed by applying it to an independent extrainstituional surgical dataset.

\section{Methods}

\section{Data source and outcomes}

The American College of Surgeons-National Surgical Quality Improvement Program (ACS-NSQIP) is a well-described and externally validated clinical database the objective of which is to assess and improve quality of care for surgical patients [23]. Trained clinical abstractors collect patientspecific, disease-related, and intra-operative variables using standardized sampling methods and definitions. Patients are identified for inclusion based on a random sample of surgical cases at participating institutions with an approximate $20 \%$ of all cases eventually included in the dataset. Further information regarding the ACS-NSQIP data structure and sampling methodology has been detailed previously [24].

The cohort of patients included in this study was similar to that used in our prior SSI model validation study [11]. Patients were identified from the institutional ACS-NSQIP sample from a prospectively maintained database of the Division of Colon and Rectal Surgery, Mayo Clinic, Rochester, Minnesota. Data analysis in this setting was authorized by the Institutional Review Board for exemption. Billing data were reviewed to determine number of diagnoses present at discharge. All non-emergent colorectal resections performed between April 2006 and June 2014 were included. Boardcertified colorectal surgeons performed all operations assisted by colorectal surgery fellows or general surgery residents. Post-operative care was provided on nursing floors that routinely handle colorectal surgery patients and standard clinical pathways were used. Patients who declined research particpation $(n=51)$ were excluded. The national, extrainstitutional ACS-NSQIP database was utilized to assess the predictive potential of the same tool built with our independent institutional data, as specified below.

\section{Outcomes}

Considering clinical and financial relevance, the primary outcome of interest was development of a deep or organ space C-SSI (according to the definition of the US Centers for Disease Control and Prevention [CDC] National Nosocomial Infection Surveillance [NNIS] criteria as any infection involving organs and spaces other than the superficial incisional compartment) [25]. Throughout the article, deep organ/space C-SSI are identified as C-OSI.

\section{Statistical analysis and handling of missing data}

A Bayesian-Probit regression model with multiple-imputation (BPMI) via Dirichlet process was used to fit a probit regression model to the outcome of C-OSI while simultaneously handling missing data. Full details of this approach are provided by Storlie et al. [26], however, an overview is given here to illustrate the main concepts.

Multiple imputation (MI), which was pioneered by Rubin [27], is an intuitively attractive concept to address this uncertainty. Multiple imputation methods typically makes the commonly misunderstood assumption of missing at random (MAR) [27]. The MAR assumption implies that the likelihood of a missing value can depend on the value of the unobserved variable, marginally, just not after accounting for all observed variables. Missing at random has been described in-depth by Schafer et al. [28]. Multiple imputation essentially aims to marginalize over the distribution of the missing data. Thus, the main caveat is that care must be taken to represent accurately the joint distribution of the complete data, i.e., that for all parameters (response and predictors) in the dataset. Most often the joint distribution of the predictors is not multivariable normal, which makes its representation difficult. The BPMI approach addresses this issue by using a flexible non-parametric density estimate for the predictor variables via the Dirichlet process [29] and latent parameter approach to allow explicitly for discrete variables. The BPMI approach also allows for certain types of missing not at random (MNAR) situations to be handled effectively by allowing for the distribution of the predictor parameters to depend on indicators of missingness for each of the parameters.

The Bayesian approach to missing data is similar to the traditional MI approach in that it aims to marginalize over the missing data and obtain the posterior distribution of the relevant parameters or predictions, conditional on only the observed data. This is typically accomplished via Markov chain Monte Carlo (MCMC) sampling [30], which is a common computational strategy to estimate the parameters of Bayesian models (see Kruschke [31]). One of the advantages of Bayesian analysis to this problem is that it provides natural measures of uncertainty in predictions via the posterior distribution. An important consequence of this to the SSI problem is that the risk prediction for a given patient is a distribution, including the uncertainty inherent in the missing predictors. Therefore, this approach allows one to ask questions such as is it possible that a given individual is at high risk but we are missing too much information to know for sure? How much would the uncertainty in risk prediction be reduced by obtaining a particular missing value? 


\section{Performance assessment}

The baseline for comparison was a conventional multivariable linear logistic regression model (generalized linear model; GLM) with indicator variables for missing data (e.g., adding a missing level to factors) and stepwise variable selection. Out-of-sample performance was evaluated with receiver operating characteristic (ROC) analysis of 10-fold cross-validated (CV) samples. Statistical analysis was performed with $\mathrm{R}$ version 3.4.3 (R Foundation for Statistical Computing, Vienna, Austria).

Both GLM and BPMI were used to predict all Mayo ClinicNSQIP observations via 10 -fold cross validation. That is, the dataset consisting of all ACS-NSQIP cases from only our instiution was partitioned into 10 subsets (folds). Each of the models was then fit 10 separate times, each time holding out the data from a different fold and using the data from the other nine folds for training. Each of these 10 models was then used to predict the observations from the corresponding fold that was held out of the training process. The final result is a set of $\mathrm{n}=2,278$ predictions for each observation in the Mayo Cliniconly dataset, each of which is an "out-of-sample" or crossvalidated prediction. These out-of-sample predictions were then used to create the ROC and related summary results described below.

As a further comparison, all ACS-NSQIP cases $(n=180,531)$ from all institutions except for ours were utilized to train the BPMI model (all ACS-NSQIP fit). The predictive potential of this externally developed model was then tested on our institutional Mayo Clinic cohort to compare its performance with the model built from only our institutional data.

\section{Results}

Among 2376 resections performed at Mayo Clinic, Rochester the C-OSI rate was $4.6 \%(n=108)$. Table 1 illustrates pre-operative demographics, laboratory values, and operative factors comparing patients who developed C-OSI and the remaining cohort.

Figure 1 illustrates the distribution of predicted risk for C-OSI among the entire cohort. A dashed line is drawn at a risk cutoff of 0.060 probability leading to C-OSI with a specificity of $80 \%$ (i.e., approximately 1 in 5 patients who do not develop C-OSI have a risk above 0.060). Figure 2 provides the confusion matrix for BPMI predictions of C-OSI using a cutoff that leads to specificity of 0.80 , i.e., a false alarm rate of approximately $20 \%$. At this level, the approach obtains a sensitivity of 0.56. The BPMI model built with the independent extrainstitutional data pool was less performant when applied on Mayo Clinic data, with a statstically significant drop to sensitivity of 0.47 (relative performance drop of 19\%).

Figure 3 displays a ROC plot using the cross-validated predictions of C-OSI risk compared with the actual events for the analytic tool (BPMI) using institutionally derived and externally derived datasets and for the standard approach (GLM FAC). The BPMI model built utilizing the institutional data pool had a cross-validated $\mathrm{AUC}=0.78$, demonstrating high predictive accuracy and substantial improvement over the standard approach. The BPMI approach trained on a much larger set of extra-institutional data also had a lower AUC $=0.74$, compared with the model fit on only our institution's data. The increased sample size comes with a cost of lower relevance (i.e., bias).
Figure 4 displays the relative parameter importance based on the posterior probability that the effect is non-zero. This figure is intended to provide a summary of the parameters that the model found to be most important to the prediction. The effect size (on the probit scale) and uncertainty in the estimated effect via $95 \%$ confidence intervals (CIs) are depicted with the plot on the far right of the figure. The plot provides an ordered list of the most influential parameters to the model. If the posterior probability equals 1.00 , this means that the parameter was included $100 \%$ of the time, i.e., the model is $100 \%$ certain that this variable should be part of the prediction. For example, American Society of Anesthesiologists (ASA) $=4$ is the top risk factor in this model with a probit regression coefficient equal 0.69 , indicating a heightened risk.

Figure 5 provides a prototype for the information to be displayed by the tool that is in development. The risk is graphically presented for a given patient, along with the factors that most contributed to an elevated risk prediction for this particular patient. The $95 \%$ prediction intervals quantify the uncertainty in the risk score due to missing factors. Missing factors that resulted in the largest proportion of this uncertainty are displayed on the right. If the uncertainty is large enough, some of the missing factors may be obtainable, e.g., certain laboratory tests, in this case albumin and serum glutamic-oxaloacetic transaminase (SGOT).

\section{Discussion}

This study revealed a high predictive value of an institutionally generic analytic tool to identify deep surgical infections in an unselected cohort of patients undergoing elective colorectal procedures in a high-volume facility, with institutional outcome data deriving from a validated national dataset. However, when fitting the same tool to the extrainstitutional ACS-NSQIP dataset, the models' predictive potential decreased substantially (performance decrease of $19 \%$ ), providing further evidence on limited applicability of externally developed predictive models [11,32]. The proposed tool attempted to address the challenge of missing data and the results validate the methodology of the suggested BPMI approach. However, the model itself has to be built with individual data out of an individual institution.

Nosocomial infections are a major driver of healthcare costs, with SSI constituting the largest portion [7,33]. The inherently high C-SSI rate after clean-contaminated colorectal surgery has been targeted for numerous quality improvement efforts, aiming to decrease rates through increased compliance to standardized infection-preventing measures [34,35]. The C-OSI represent a particular challenge because of non-specific clinical presentation, an extended length of stay, and increased mortality [36]. Recent large surveillance studies found that only $47 \%$ of C-OSI were detected by post-operative day seven and that more than $80 \%$ needed interventional source control [37,38]. A large European multicenter trial revealed a C-OSI treatment failure rate of more than $34 \%$, with treatment failure defined as signs and symptoms of SSI or death at 30 days postsurgery [37]. Taken together, these results emphasize the importance of timely detection and treatment of C-OSI because of the implications of treatment delays.

Accurate prediction of post-operative C-OSI may prevent deterioriation and dispersion of a latent infection at an earlier stage and allow for prompt management during the index 
Table 1. Pre-Operative and Operative Factors of Deep Organ/Space Surgical Site Infection

\begin{tabular}{|c|c|c|c|c|}
\hline & $\begin{array}{c}\text { No } C-O S I \\
\mathrm{n}=2268\end{array}$ & $\begin{array}{c}C-O S I \\
\mathrm{n}=108\end{array}$ & $\begin{array}{c}\text { Total } \\
\mathrm{n}=2367\end{array}$ & $\mathrm{p}$ \\
\hline Age, median (IQR) & $58(44,70)$ & $50(31,67)$ & $58(44,70)$ & $<\mathbf{0 . 0 0 1}$ \\
\hline \multicolumn{5}{|l|}{ Age, category } \\
\hline$<40$ & $443(19.5)$ & $37(34.3)$ & $480($ ( 20.2) & \\
\hline $40-54$ & $511(22.5)$ & $22(20.4)$ & 533 ( 22.4) & \\
\hline $55-64$ & $476(21)$ & $19(17.6)$ & 495 ( 20.8) & \\
\hline $65-74$ & 473 ( 20.9) & $18(16.7)$ & 491 ( 20.7) & \\
\hline $75+$ & $365(16.1)$ & $12(11.1)$ & 377 ( 15.9) & \\
\hline \multicolumn{5}{|l|}{ Sex } \\
\hline Male & $1162(51.2)$ & $57(52.8)$ & $1219(51.3)$ & 0.830 \\
\hline \multicolumn{5}{|l|}{ BMI* } \\
\hline $30+$ & $618(27.3)$ & $34(31.5)$ & $652(27.5)$ & 0.403 \\
\hline Race & & & & 0.053 \\
\hline Non-Hispanic white & $2,000(88.2)$ & $88(81.5)$ & $2,088(87.9)$ & \\
\hline Other or unknown & $268(11.8)$ & $20(18.5)$ & $288(12.1)$ & \\
\hline ASA & & & & 0.219 \\
\hline 1 or 2 & $1,526(67.3)$ & $66(61.1)$ & $1592(67)$ & \\
\hline 3 or 4 & $742(32.7)$ & $42(38.9)$ & $784(33)$ & \\
\hline \multicolumn{5}{|l|}{ Hypertension requiring medication } \\
\hline Yes & 803 ( 35.4$)$ & $30(27.8)$ & $833(35.1)$ & 0.129 \\
\hline $\begin{array}{l}\text { Steroid use for chronic condition } \\
\text { Yes }\end{array}$ & $358(15.8)$ & $32(29.6)$ & $390(16.4)$ & $<\mathbf{0 . 0 0 1}$ \\
\hline \multicolumn{5}{|l|}{ Current smoker } \\
\hline Yes & $270(11.9)$ & $22(20.4)$ & $292(12.3)$ & 0.014 \\
\hline \multicolumn{5}{|l|}{ Diabetes mellitus } \\
\hline Yes & $193(8.5)$ & $22(20.4)$ & $215(9.1)$ & $<\mathbf{0 . 0 0 1}$ \\
\hline \multicolumn{5}{|l|}{ SIRS/sepsis/septic shock ${ }^{\mathrm{a}}$} \\
\hline Yes & $55(2.4)$ & $10(9.4)$ & $65(2.8)$ & $<0.001$ \\
\hline Wound classification & & & & $<\mathbf{0 . 0 0 1}$ \\
\hline Clean, clean/contaminated & $2,035(89.7)$ & $79(73.1)$ & $2114(89)$ & \\
\hline Contaminated & $104(4.6)$ & $5(5.6)$ & $110(4.6)$ & \\
\hline Dirty/Infected & $129(5.7)$ & $23(21.3)$ & $152(6.4)$ & \\
\hline \multicolumn{5}{|l|}{ Disseminated cancer } \\
\hline Yes & $104(4.6)$ & $9(8.3)$ & $113(4.8)$ & 0.120 \\
\hline \multicolumn{5}{|l|}{ Pre-operative $\mathrm{WBC}^{\mathrm{a}}$} \\
\hline \multicolumn{4}{|l|}{ Pre-operative hematocrit ${ }^{\mathrm{a}}$} & 0.003 \\
\hline$<38$ & $973(46.6)$ & $57(58.8)$ & $1,030(47.1)$ & 0.025 \\
\hline \multicolumn{5}{|l|}{ Pre-operative serum albumin ${ }^{a}$} \\
\hline$<3.5$ & $178(16.1)$ & $19(32.2)$ & $197(16.9)$ & 0.002 \\
\hline \multicolumn{5}{|l|}{ Pre-operative SGOT $^{\mathrm{a}}$} \\
\hline $40+$ & $142($ 8.6) & $8(11.6)$ & $150(8.7)$ & 0.520 \\
\hline \multicolumn{4}{|l|}{ Diagnosis } & 0.005 \\
\hline Colon cancer & 504 ( 22.2) & $14(13)$ & $518(21.8)$ & \\
\hline Rectal cancer & $366(16.1)$ & $19(17.6)$ & $385(16.2)$ & \\
\hline Ulcerative colitis & $348(15.3)$ & $26(24.1)$ & $374(15.7)$ & \\
\hline Crohn's & $240(10.6)$ & $19(17.6)$ & $259(10.9)$ & \\
\hline Diverticular disease & 299 ( 13.2) & $8(7.4)$ & 307 ( 12.9) & \\
\hline Other & $511(22.5)$ & $22(20.4)$ & $533(22.4)$ & \\
\hline \multicolumn{4}{|l|}{ Operative approach } & $<0.001$ \\
\hline Laparascopic & $1225(54)$ & $31(28.7)$ & $1,256(52.9)$ & \\
\hline Open & $1034(46)$ & $77(71.3)$ & $1120(47.1)$ & \\
\hline Operative time, median (IQR) & $185(134,248)$ & $222(158,281)$ & $186(136,249)$ & $<\mathbf{0 . 0 0 1}$ \\
\hline \multicolumn{5}{|l|}{ Operative time, category } \\
\hline$\leq 2 \mathrm{~h} 14 \mathrm{~min}$ & $572(25.2)$ & $11(10.2)$ & $538(24.5)$ & \\
\hline $2 \mathrm{~h} 15 \mathrm{~min}$ to $2 \mathrm{~h} 59 \mathrm{~min}$ & 495 ( 21.8) & $28(25.9)$ & $523(22)$ & \\
\hline $3 \mathrm{~h}$ to $3 \mathrm{~h} 59 \mathrm{~min}$ & $582(25.7)$ & $24(22.2)$ & $606(25.5)$ & \\
\hline$\geq 4 \mathrm{~h}$ & 619 ( 27.3) & $45(41.7)$ & 664 ( 27.9) & \\
\hline
\end{tabular}

(continued) 
TABle 1. (CONTINUED)

\begin{tabular}{|c|c|c|c|c|}
\hline & $\begin{array}{c}\text { No C-OSI } \\
\mathrm{n}=2268\end{array}$ & $\begin{array}{c}C-O S I \\
\mathrm{n}=108\end{array}$ & $\begin{array}{c}\text { Total } \\
\mathrm{n}=2367\end{array}$ & $\mathrm{p}$ \\
\hline \multicolumn{5}{|l|}{ Stoma, any } \\
\hline Yes & $973(42.9)$ & $68(63)$ & $1,041(43.8)$ & $<\mathbf{0 . 0 0 1}$ \\
\hline Stoma, type & & & & $<\mathbf{0 . 0 0 1}$ \\
\hline Ileostomy & $725(74.5)$ & $48(70.6)$ & $773(74.3)$ & \\
\hline Colostomy & $248(25.5)$ & $20(29.4)$ & $268(26.2)$ & \\
\hline Procedure type & & & & $<0.001$ \\
\hline Right & $655(28.9)$ & $18(16.7)$ & $673(28.3)$ & \\
\hline Left & $131(5.8)$ & $8(7.4)$ & $139(5.9)$ & \\
\hline Left with rectal & $705(31.1)$ & $20(18.5)$ & $725(30.5)$ & \\
\hline Total & $259(11.4)$ & $18(16.7)$ & 277 ( 11.7) & \\
\hline Total with rectal & $44(1.9)$ & $3(2.7)$ & $47(2)$ & \\
\hline Rectal & $112(4.9)$ & $12(11.1)$ & $124(5.2)$ & \\
\hline IPAA & $156(6.9)$ & $14(13)$ & $170(7.1)$ & \\
\hline APR & $206(9.1)$ & $15(13.9)$ & $221(9.3)$ & \\
\hline Total RVU, Median (IQR) & $35.7(30,49)$ & $37.1(32.8,54.1)$ & $35.7(30,49.2)$ & 0.009 \\
\hline
\end{tabular}

Bold values indicate significant values $(\mathrm{p}<0.05)$.

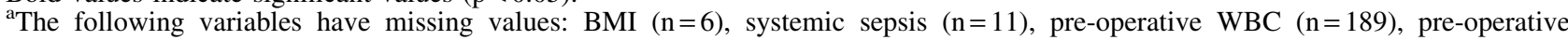
hematocrit $(n=190)$, pre-operative serum albumin $(n=1,211)$, pre-operative SGOT $(n=657)$.

$\mathrm{C}$-OSI = deep organ/space infection; BMI = body mass index; ASA = American Society of Anesthesiologists; SIRS = systemic inflammatory response syndrome; $\mathrm{WBC}=$ white blood cell count; $\mathrm{SGOT}=$ serum glutamic-oxaloacetic transaminase; $\mathrm{RVU}=$ relative value units .

hospitalization. Several C-SSI predictive models of varying complexity in the pre- and post-operative setting have been suggested. Gervaz et al. [14] developed a clinical score through multivariable logistic regression of risk factors and assessed discriminative ability through ROC analysis. Despite ease of use and internal validation, a recent validation study demonstrated limited validity of the score when applied in an independent dataset [11]. This was mainly explained by institution-specific factors as drivers for C-SSI that cannot be extrapolated to other institutions. This latter study revealed also a lack of ability of two national assessment models

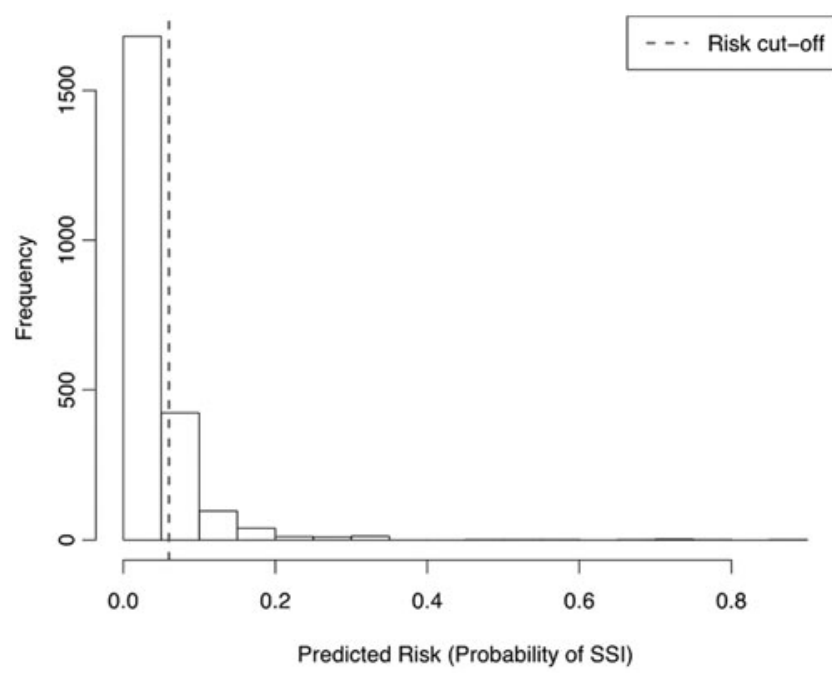

FIG. 1. Distribution of predictive risk for C-OSI among the entire cohort. The dashed line indicates the set cut-off value of probaility $=0.060$ leading to a $20 \%$ false alarm rate. $\mathrm{C}$-OSI = colorectal deep organ/space infection; SSI = surgical site infection.
(ACS-NSQIP- and NNIS-based) to predict SSI in the independent dataset, similar to the present study. Arguably, both scores were developed with historical data before changes in widely adopted peri-operative care strategies (i.e., enhanced recovery principles or oral antibiotic agents with mechanical bowel preparation) occurred $[39,40]$.

Disparity in accuracy of initial C-SSI assessment represents an additional challenge when comparing different predictive models. Even though a large variety of surveillance methods has been described, studies assessing comparative reliability of different assessment tools are still scarce $[41,42]$. This is critical and questions universal applicability of predictive risk models.

Our tool attempted to account for more homogeneity by specific targeting of clinically relevant C-OSI and to overcome methodological shortcomings through a novel statistical approach to handle missing data, which constitute an inherent drawback when dealing with heldout data. Interestingly, the score demonstrated high predictive accuracy and substantial improvement over the standard approach. However, the tools' performance decreased when built on extra-institutional independent data to reach similar performance as the standard approach (green and red lines in Figure 3). In other words, the model itself is not transferable to other institutions and has to be built with individual data of a specific institution, however, the BPMI approach to build an insitution specific model is generally applicable.

Arguably, the score calculation by the model is complex and application in clinical practice is not as straightforward as it is with other scores based on simple rules or linear combinations, as illustrated in the rather complex representation of variable importance estimates in Figure 4. The complexity of the score reflects both the multifactorial and complex pathogenesis of C-SSI and thorough statistical methodology. This concern can be addressed by implementing the tool into clinical practice as an online application, that automatically gathers the relevant data from the electronic medical record, 


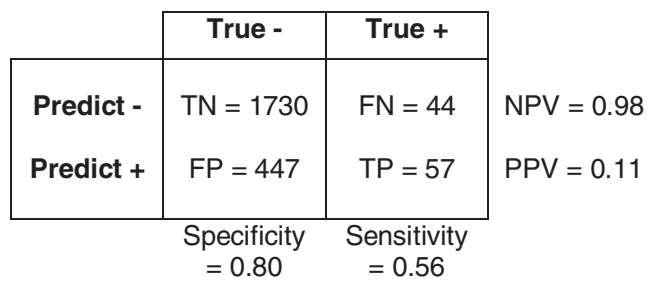

\begin{tabular}{|c|c|c|c|}
\hline & True - & True + & \\
\hline Predict - & $\mathrm{TN}=1730$ & $\mathrm{FN}=54$ & $\mathrm{NPV}=0.97$ \\
\hline Predict + & $\mathrm{FP}=447$ & $\mathrm{TP}=47$ & $P P V=0.10$ \\
\hline & $\begin{array}{l}\text { Specificity } \\
=0.80\end{array}$ & $\begin{array}{l}\text { Sensitivity } \\
=0.47\end{array}$ & \\
\hline
\end{tabular}

FIG. 2. Confusion matrix based on 10-fold cross validation for the proposed approach (BPMI) for predicting deep/organ space surgical site infection (C-OSI) at a 10\% false alarm threshold (i.e., setting specificity $=0.90)$. BPMI $=$ Bayesian-Probit regression model with multiple-imputation; $\mathrm{C}-\mathrm{OSI}=$ colorectal deep/organ space infection; $\mathrm{TN}=$ true-negative; $\mathrm{FN}=$ falsenegative; $\mathrm{FP}=$ false-positive; $\mathrm{TP}=$ true-positive; $\mathrm{NPV}=$ negative predictive value; $\mathrm{PPV}=$ positive predictive value.

computes the score and variable importance measures, and presents this information to the clinican as shown in Figure 5. In collaboration with biostatisticians and IT personnel, creating a tool using the suggested approach is feasible in any institution, because the model is implemented in the $\mathrm{R}$ language [43]. As a result, obtaining missing factors, e.g., specific laboratory tests, will help to improve the accuracy of risk prediction. Once implemented, the score may thus ultimately provide guidance in daily clinical practice. The predicted C-OSI risk may facilitate the clinicians' decision how to intervene and whether to undertake further diagnostic or therapeutic steps during the index hospital stay potentially preventing costly hospital re-admissions. This includes increased physician awareness toward infectious symptoms, signs, and parameter, and either pre-dismissal education and close follow-up for high-risk patients, or imaging prior to discharge.
The cutoff used in the results of this study of approximately 0.06 probability (leading to $80 \%$ specificity) is merely a suggestion and was chosen because it results in $80 \%$ specificity. The actual cutoff to be used in practice would have to depend on the actual intervention to be used and the capacity for conducting whatever additional tests and follow-up that this might entail. The decision whether to delay patient discharge or to proceed with workup ultimately relies on clinical pragmatism. The next step in this line of research is a silent pilot study to evaluate the model's performance and useability in a true prospective production setting. Finally, a cluster-randomized trial (e.g., stepped wedge approach across surgeons) would be needed to assess the tool's effectiveness in altering clinical outcomes (e.g., re-admission, length of stay, cost, etc.). Therefore, the results of this study should not be considered as final but as preliminary results of work in

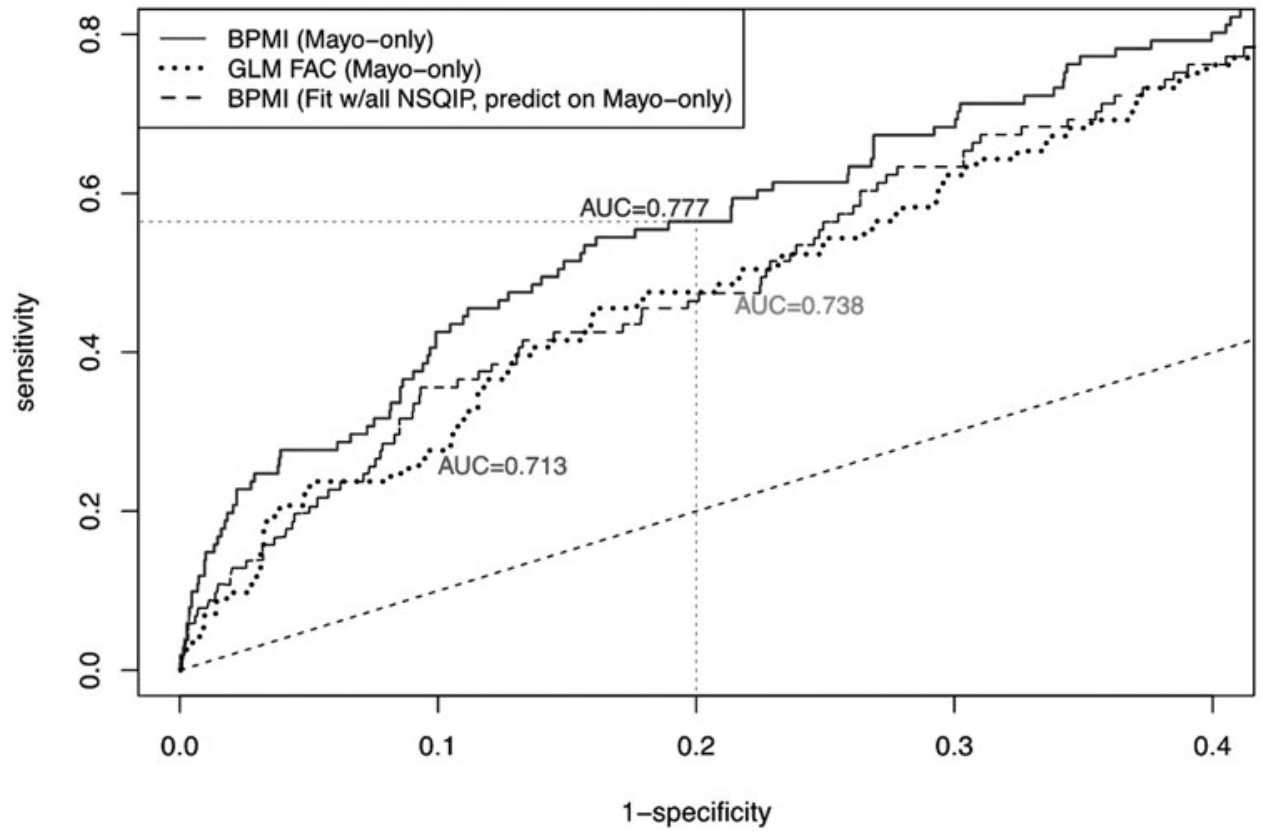

FIG. 3. The ROC based on 10-fold cross-validation for the proposed approach (BPMI) and the standard approach (GLM FAC) for predicting deep organ/space surgical site infection. The ROC curves of the BPMI model utilizing the Mayo Cliniconly data pool (solid line), the BPMI model utilizing the national NSQIP data pool to predict C-OSI on Mayo-Clinic only data (dashed line), and the GLM FAC model built on Mayo Clinic-only data (dotted line). ROC=receiver operator characteristic; $\mathrm{BPMI}=$ Bayesian-Probit regression model with multiple-imputation; GLM FAC = stepwsie logistic regression treating missingness with factor/indicator parameters; C-OSI = colorectal deep organ/space infection; AUC = area under curve. 


$\begin{array}{lrr}\text { Variable } & \text { Posterior } & \text { Estimate } \\ \text { ASA }=4 & 1.00 & 0.69 \\ \text { DM2 } & 1.00 & 0.53 \\ \text { DM1 } & 1.00 & 0.50 \\ \text { Days After Surgery } & 1.00 & 0.31 \\ \text { Age } & 1.00 & -0.23 \\ \text { Wound=3/4 } & 0.99 & 0.57 \\ \text { PT Lab } & 0.97 & 0.33 \\ \text { Race=Native } & 0.94 & 0.99 \\ \text { INR Lab } & 0.83 & -0.19 \\ \text { Steroids } & 0.64 & 0.22 \\ \text { Open } & 0.61 & 0.20 \\ \text { Revascularization } & 0.53 & 0.31 \\ \text { Ascites } & 0.45 & 0.19 \\ \text { >=1 Days Before Surgery } & 0.44 & 0.12 \\ \text { Chemo } & 0.38 & 0.11 \\ \text { Smoker } & 0.37 & 0.07\end{array}$

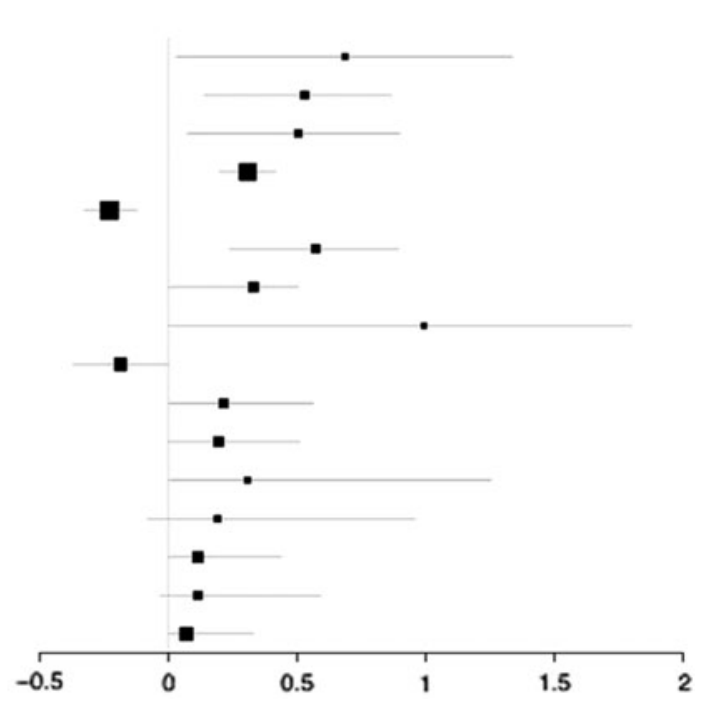

FIG. 4. Parameter importance summary for all predictors with a posterior inclusion probability above 0.3 . Representation of clinical variables and their relative variable importance. Calculations are based on the posterior probability that the effect is non-zero. Posterior is the posterior probability that the parameter was included in the model. Estimate is the posterior mean estimate of the coeficient in the probit model. Ninety-five percent confidence intervals along with estimate are presented graphically on the far right. Only parameters with a posterior inclusion probability above 0.3 are displayed. ASA = American Society of Anesthesiologists; DM=diabetes mellitus; wound= wound class type 3 (contaminated) and 4 (infectious); $\mathrm{PT}=$ prothrombin; INR = international normalized ratio.

progress, with a potential to overcome some of the shortcomings encountered with modeling in clinical surgery.

Several limitations of this study need to be addressed. First, the analytic tool was developed on institutional heldout data harboring inherent limitations of retrospective data collection. The data were derived from a high-volume tertiary care center, hence, this approach should primarily be applied in similar settings with high patient accrual. Local disparities need to be considered, as shown by discrepancies in performance according to the data pool utilized for fitting of the model. Furthermore, it has to be considered that ACS-NSQIP harbors only approximately $20 \%$ of surgical cases, however,

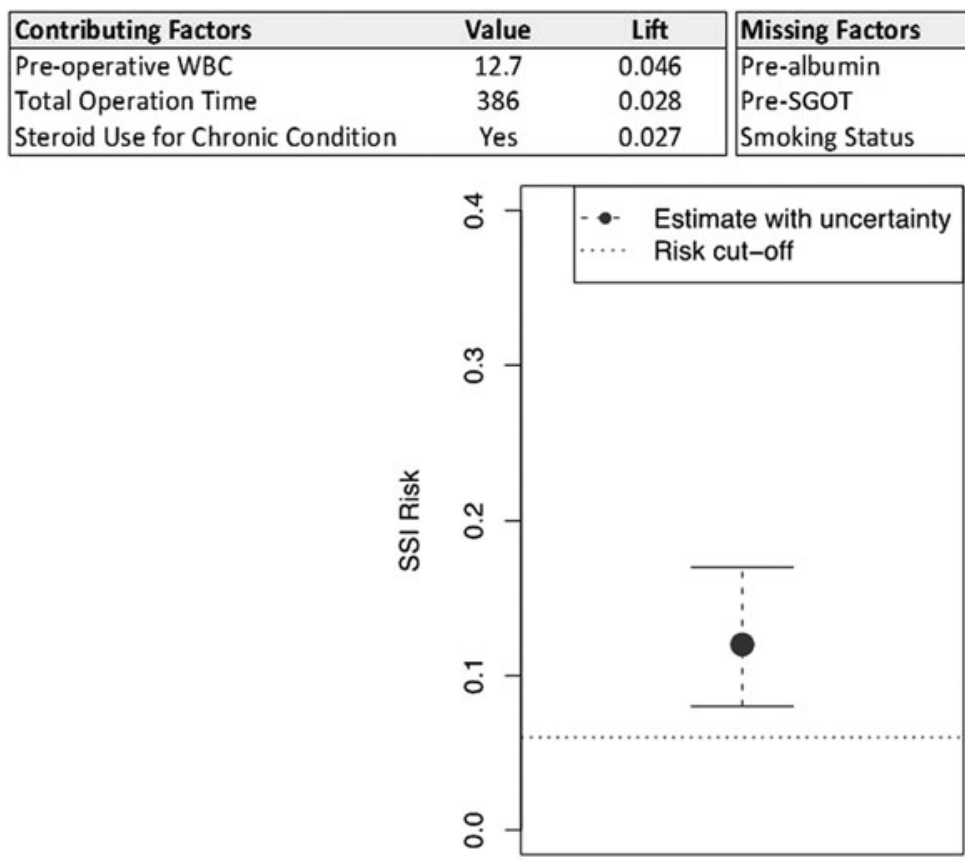

FIG. 5. Proposed monitoring tool display. The risk of SSI along with uncertainty limits are graphically presented, along with the factors that most contributed to an elevated risk prediction. The $95 \%$ prediction band quantifies the uncertainty in the risk score because of missing factors. Missing factors that resulted in the largest proportion of this uncertainty are displayed on the right. If the uncertainty is large enough, some of the missing factors may be obtainable, e.g., certain laboratory tests. $\mathrm{WBC}=$ white blood cell count; $\mathrm{SGOT}=$ serum glutamic-oxaloacetic transaminase. 
representing standardized assessement, repeatedly validated data pool, and high interobserver reliability [24]. Second, the tool depends on quality of C-SSI surveillance; standardized C-SSI definitions with precise criteria are of utmost importance especially in post-discharge surveillance. Third, modifications in surgical and peri-operative care (enhanced recovery, implementation of SSI prevention bundle) inevitably occurred over the 12-year study period. However, at the Mayo Clinic, procedures and care were highly standardized throughout the inclusion period. Fourth, the tool provides a predictive score upon the conclusion of surgery and does not consider temporal aspects, nor provide information when the infection occurs. Post-operative patient monitoring and inclusion of dynamic items such as vitals, post-operative laboratory levels, and length of stay could provide additional information to refine the tool. Furthermore, temporal aspects may be refined using Bayesian Cox proportional hazard regression or machine learning with Bayes classifiers to incorporate time-dependent observations [44]. This could be of particular relevance in the decision for discharge, weighing longer hospitalizations with risk of re-admission, with consequences for all stakeholders on either side. Finally, the algorithm needs prospective validation to assess performance in clinical practice before widespread implementation.

\section{Conclusions}

The institutionally generic analytic tool identified a substantial proportion of patients with C-OSI correctly and may help to improve outcomes and reduce costs through early identification and management. However, fitting the tool with external, independent data decreased its predictive potential, emphasizing the importance of considering institutionspecific standards and definitions when building predictive algorithms. Taken together, the new approach to missing data may allow refining risk prediction, providing models are built with outcome data of individual institutions.

\section{Acknowledgments}

The authors gratefully acknowledge the support of the Mayo Clinic Department of Surgery and the Robert D. and Patricia E. Kern Center for the Science of Health Care Delivery as substantial contributors of in-kind resources to the project. Additionally, Dr. Bergquist acknowledges the support of the Mayo Clinic Clinician Investigator Training Program for their salary support. Drs. Habermann, Storlie \& Etzioni receive and gratefully acknowledge salary support from the Robert D. and Patricia E. Kern Center for the Science of Health Care Delivery at the Mayo Clinic. Finally, we would like to thank the Academic Surgical Congress for affording us the opportunity to present this work at their annual meeting in February 2017. Dr. Grass was generously supported by the Société Académique Vaudoise, Lausanne, Switzerland and by the SICPA foundation, Lausanne, Switzerland.

This manuscript has not previously or concurrently been submitted for publication. The work has been presented at the annual meeting of the Academic Surgical Congress in February 2017.

\section{Funding Information}

The Mayo Clinic Robert D. and Patricia E. Kern Center for the Science of Health Care Delivery provides salary support for Dr. Habermann, Dr. Storlie, and Dr. Etzioni and in kind material support for Dr. Bergquist. Dr. Bergquist has received salary support from the Mayo Clinic Clinician Investigator Training program.

\section{Author Disclosure Statement}

All authors have no conflict of interest or competing interests to declare.

\section{References}

1. Penn CA, Kamdar NS, Morgan DM, et al. Preoperatively predicting non-home discharge after surgery for gynecologic malignancy. Gynecol Oncol 2019;152:293-297.

2. Simoes CM, Carmona MJC, Hajjar LA, et al. Predictors of major complications after elective abdominal surgery in cancer patients. BMC Anesthesiol 2018;18:49.

3. Al-Homoud S, Purkayastha S, Aziz O, et al. Evaluating operative risk in colorectal cancer surgery: ASA and POSSUMbased predictive models. Surg Oncol 2004;13:83-92.

4. Ferjani AM, Griffin D, Stallard N, Wong LS. A newly devised scoring system for prediction of mortality in patients with colorectal cancer: A prospective study. Lancet Oncol 2007;8:317-322.

5. Hechenbleikner EM, Hobson DB, Bennett JL, Wick EC. Implementation of surgical quality improvement: Auditing tool for surgical site infection prevention practices. Dis Colon Rectum 2015;58:83-90.

6. Smith RL, Bohl JK, McElearney ST, et al. Wound infection after elective colorectal resection. Ann Surg 2004;239:599605.

7. de Lissovoy G, Fraeman K, Hutchins V, et al. Surgical site infection: Incidence and impact on hospital utilization and treatment costs. Am J Infect Control 2009;37: 387-397.

8. Rajaram R, Chung JW, Kinnier CV, et al. Hospital characteristics associated with penalties in the centers for Medicare \& Medicaid Services hospital-acquired condition reduction program. JAMA 2015;314:375-383.

9. Thompson KM, Oldenburg WA, Deschamps C, et al. Chasing zero: The drive to eliminate surgical site infections. Ann Surg 2011;254:430-436.

10. Polk HC Jr, Lopez-Mayor JF. Postoperative wound infection: A prospective study of determinant factors and prevention. Surgery 1969;66:97-103.

11. Bergquist JR, Thiels CA, Etzioni DA, et al. Failure of colorectal surgical site infection predictive models applied to an independent dataset: Do they add value or just confusion? J Am Coll Surg. 2016;222(4):431-8.

12. Geubbels EL, Grobbee DE, Vandenbroucke-Grauls CM, et al. Improved risk adjustment for comparison of surgical site infection rates. Infect Control Hosp Epidemiol 2006; 27:1330-1339.

13. Cima RR, Bergquist JR, Hanson KT, Thiels CA, Habermann EB. Outcomes are local: Patient, disease, and procedurespecific risk factors for colorectal surgical site infections from a single Institution. J Gastrointest Surg 2017;21:1142-1152.

14. Gervaz P, Bandiera-Clerc C, Buchs NC, Eisenring MC, Troillet N, Perneger T, et al. Scoring system to predict the risk of surgical-site infection after colorectal resection. Br J Surg 2012;99:589-595.

15. Sanger PC, van Ramshorst GH, Mercan E, et al. A Prognostic model of surgical site infection using daily clinical wound assessment. J Am Coll Surg 2016;223:259-270. 
16. Takakura $\mathrm{Y}$, Hinoi $\mathrm{T}$, Egi $\mathrm{H}$, et al. Procalcitonin as a predictive marker for surgical site infection in elective colorectal cancer surgery. Langenbecks Arch Surg 2013;398: 833-839.

17. Woelber E, Schrick EJ, Gessner BD, Evans HL. Proportion of surgical site infections occurring after hospital discharge: A systematic review. Surg Infect 2016;17:510-519.

18. Damle RN, Cherng NB, Flahive JM, et al. Clinical and financial impact of hospital readmissions after colorectal resection: Predictors, outcomes, and costs. Dis Colon Rectum 2014;57:1421-1429.

19. Richter V, Cohen MJ, Benenson S, et al. Patient selfassessment of surgical site infection is inaccurate. World $\mathrm{J}$ Surg 2017;41:1935-1942.

20. Ady J, Fong Y. Imaging for infection: From visualization of inflammation to visualization of microbes. Surg Infect 2014;15:700-707.

21. Sterne JA, White IR, Carlin JB, et al. Multiple imputation for missing data in epidemiological and clinical research: Potential and pitfalls. BMJ 2009;338:b2393.

22. Pedersen AB, Mikkelsen EM, Cronin-Fenton D, et al. Missing data and multiple imputation in clinical epidemiological research. Clin Epidemiol 2017;9:157-166.

23. Ingraham AM, Richards KE, Hall BL, Ko CY. Quality improvement in surgery: The American College of Surgeons National Surgical Quality Improvement Program approach. Adv Surg 2010;44:251-267.

24. Shiloach M, Frencher SK Jr, Steeger JE, et al. Toward robust information: Data quality and inter-rater reliability in the American College of Surgeons National Surgical Quality Improvement Program. J Am Coll Surg 2010;210:6-16.

25. Emori TG, Culver DH, Horan TC, et al. National nosocomial infections surveillance system (NNIS): Description of surveillance methods. Am J Infect Control 1991;19:19-35.

26. Storlie C, Therneau T, Carter R, et al. Prediction and Inference with missing data in patient alert systems. J Am Stat Assoc 2020;115:32-46.

27. Rubin DB. Inference and missing data. Biometrika 1976; 63:581-592.

28. Schafer JL, Graham JW. Missing data: Our view of the state of the art. Psychol Methods 2002;7:147-177.

29. Storlie CB, Myers SM, Katusic SK, et al. Clustering and variable selection in the presence of mixed variable types and missing data. Stat Med 2018;37:2884-2899.

30. Bijak J, Bryant J. Bayesian demography 250 years after Bayes. Popul Stud (Camb) 2016;70:1-19.

31. Kruschke JK. Doing Bayesian Data Analysis: A Tutorial with R, JAGS, and Stan, 2nd ed. London: Academic Press, 2014.

32. Cima RR, Hassan I, Poola VP, et al. Failure of institutionally derived predictive models of conversion in laparoscopic colorectal surgery to predict conversion outcomes in an independent data set of 998 laparoscopic colorectal procedures. Ann Surg 2010;251:652-658.

33. Wick EC, Shore AD, Hirose K, et al. Readmission rates and cost following colorectal surgery. Dis Colon Rectum 2011; 54:1475-1479.
34. Keenan JE, Speicher PJ, Thacker JK, et al. The preventive surgical site infection bundle in colorectal surgery: An effective approach to surgical site infection reduction and health care cost savings. JAMA Surg 2014;149:10451052.

35. Cima R, Dankbar E, Lovely J, et al. Colorectal surgery surgical site infection reduction program: A national surgical quality improvement program-driven multidisciplinary single-institution experience. J Am Coll Surg 2013;216: 23-33.

36. Shaw E, Gomila A, Piriz M, et al. Multistate modelling to estimate excess length of stay and risk of death associated with organ/space infection after elective colorectal surgery. J Hosp Infect 2018;100:400-405.

37. Gomila A, Carratala J, Camprubi D, et al. Risk factors and outcomes of organ-space surgical site infections after elective colon and rectal surgery. Antimicrob Resist Infect Control 2017;6:40.

38. Martin D, Hubner M, Moulin E, et al. Timing, diagnosis, and treatment of surgical site infections after colonic surgery: Prospective surveillance of 1263 patients. J Hosp Infect 2018; 100:393-3990.

39. Rollins KE, Javanmard-Emamghissi H, Lobo DN. Impact of mechanical bowel preparation in elective colorectal surgery: A meta-analysis. World J Gastroenterol 2018;24: 519-536.

40. Moonesinghe SR, Grocott MPW, Bennett-Guerrero E, et al. American Society for Enhanced Recovery (ASER) and Perioperative Quality Initiative (POQI) joint consensus statement on measurement to maintain and improve quality of enhanced recovery pathways for elective colorectal surgery. Perioper Med (Lond) 2017;6:6.

41. Lyman WB, Passeri M, Cochran A, et al. Discrepancy in postoperative outcomes between auditing databases: A NSQIP comparison. Am Surg 2018;84:1294-1298.

42. Petherick ES, Dalton JE, Moore PJ, Cullum N. Methods for identifying surgical wound infection after discharge from hospital: A systematic review. BMC Infect Dis 2006; 6:170.

43. Li Y. Using the open-source statistical language $\mathrm{R}$ to analyze the dichotomous Rasch model. Behav Res Methods 2006;38:532-541.

44. Chen Q, Wu H, Ware LB, Koyama T. A Bayesian approach for the Cox proportaional hazards model with covariates subject to detection limit. Int J Stat Med Res 2014;3:32-43.

Address correspondence to: Dr. Robert R. Cima Division of Colon and Rectal Surgery Mayo Clinic

200 First Street Southwest Rochester, MN 55905

USA

E-mail: Cima.robert@mayo.edu 